\title{
Semantic Abilities in Children With Pragmatic Language Impairment: The Case of Picture Naming Skills
}

\author{
Mieke Pauline Ketelaars \\ Suzanne Irene Alphonsus Hermans \\ Radboud University Nijmegen, \\ Behavioural Science Institute, \\ the Netherlands \\ Juliane Cuperus \\ Kino Jansonius \\ Sint Marie Eindhoven, \\ the Netherlands \\ Ludo Verhoeven \\ Radboud University Nijmegen, \\ Behavioural Science Institute, \\ the Netherlands
}

Purpose: The semantic abilities of children with pragmatic language impairment (PLI) are subject to debate. The authors investigated picture naming and definition skills in 5-year-olds with PLI in comparison to typically developing children.

Method: 84 children with PLI and 80 age-matched typically developing children completed receptive vocabulary, picture naming, and definition tasks.

Results: The PLI group scored lower on the receptive vocabulary and picture naming tasks. Word length and frequency affected naming accuracy in both groups. Children with PLI showed higher numbers of semantic errors, nonrelated errors, and omissions and circumlocutions. The error-type distribution differed between groups: PLI children showed disproportionate levels of nonrelated errors. In the definition task, PLI children showed lower information accuracy for accurately named pictures and comparable accuracy for incorrectly named pictures. Qualitative analysis suggested a high incidence of pragmatically inappropriate definitions for the PLI group. Naming accuracy for both groups improved equally after giving semantic cues.

Conclusions: These findings suggest a deficit in object identification and/or naming selection. It might be premature to conclude that children with PLI show normal semantic abilities. The results are largely consistent with a general language delay; however, there is also some evidence of a qualitative difference between both groups.

KEY WORDS: pragmatic language impairment, naming accuracy, error type, definition accuracy, semantic cues

C hildren with pragmatic language impairment (PLI) are characterized by problems understanding and applying social rules in language (Bishop, 2000; Van Balkom \& Verhoeven, 2004). Although this is its distinguishing characteristic, PLI has also been associated with subtle semantic problems (e.g., Botting \& Adams, 2005). The exact nature of these semantic problems and their underlying mechanisms are still subject to debate (Botting \& Conti-Ramsden, 1999). In the present article, we investigated semantic abilities in children with PLI using a picture naming and definition task. This design may shed more light on the exact symptoms experienced by these children in the area of semantic problems.

Early studies reporting on pragmatic language problems used the term semantic pragmatic language syndrome (Bishop \& Adams, 1989). The term was used to describe children with language disorders who, despite normal language form, showed both semantic and pragmatic problems. The semantic problems consisted of unusual word choice and word-finding difficulties; the pragmatic problems consisted of impaired conversational abilities. The symptoms exhibited by this group of children were classified as a subtype of specific language impairment (SLI). 
However, this classification is much disputed since the term has come into use (e.g., Brook \& Bowler, 1992). Discussion on the validity of the classification has mainly been caused by the symptom resemblance of PLI with autism spectrum disorders such as pervasive developmental disorder-not otherwise specified and Asperger's syndrome. Regardless of the diagnostic validity of PLI as a separate disorder or as a subclassification of a language disorder, pragmatic language problems constitute one of the core symptoms of individuals with autism (Miniscalco, Hagberg, Kadesjö, Westerlund, \& Gillberg, 2007). In addition, pragmatic language problems have been found in children with attention-deficit/hyperactivity disorder (ADHD; e.g., Camarata \& Gibson, 1999; Geurts et al., 2004).

More recently, some researchers have suggested that semantic problems are not a necessary symptom of PLI (Bishop, 1998) based on the finding that a group of children with PLI within a sample of 7-year-old children with SLI did not differ in their scores on a picture naming task from a group of 7-year-old children with SLI. As such, children with PLI showed similar semantic abilities compared with children with SLI. The findings of this study have led to the recent adoption of the term $P L I$ as a replacement of the original term and to debate on whether or not PLI should be regarded as a subtype of SLI. However, we question the validity of eliminating semantic problems as a symptom on the basis of several arguments. First, we question whether comparable semantic abilities of children with SLI and children with PLI can be used as a valid argument to eliminate semantic problems as a symptom of PLI. Children with SLI are known to experience semantic problems (Brackenbury \& Pye, 2005; McGregor, Newman, Reilly, \& Capone, 2002), and if the research shows that children with PLI perform at a similar level, then this implies that children with PLI experience semantic problems as well. The elimination of semantic problems as a symptom may, unjustifiably, lead clinicians to assume that semantic abilities are generally unaffected. In order to come to this conclusion, research first has to show that children with PLI do not show semantic problems in comparison to typically developing (TD) children. If they do indeed show semantic problems, then the second step would be to assess how the semantic abilities of children with PLI compare with those of children with SLI. Furthermore, it should be noted that in the studies conducted so far, children with PLI have been investigated in an identified SLI group. This might have resulted in a restricted view on PLI and may misrepresent the relationship between SLI and PLI.

There are several researchers who report on semantic problems in children with PLI. For instance, Rapin and Allen (1983) name word-finding difficulties as one of the core symptoms in children with what was then called semantic-pragmatic disorder. In addition, Sahlén and
Nettelbladt (1993) report on two longitudinal case studies in which they found prolonged impaired picture naming. They suggest that pragmatic deficits might occur as a secondary consequence of semantic problems. The two children who were followed in Sahlén and Nettelbladt showed low picture naming accuracy and a high rate of semantic errors. In addition, one of the children seemed to exhibit considerable lexical retrieval problems as evidenced by large stimulus-response times. Other evidence of semantic problems comes from studies by Bishop and Adams (1989) and Bishop and Norbury (2002), who found a high rate of strange words and stereotyped language in children with PLI. More recently, Botting and Adams (2005) found lower performance on a similar meaning task, in which the children were shown a word and subsequently had to choose a word of similar meaning, for 11-year-old children with PLI compared with agematched peers and language-matched younger peers.

In order to study the semantic abilities of children with PLI in relative isolation of other (socio)linguistic skills, we used a picture naming task in the present study. In picture naming tasks, both naming accuracy and the pattern of naming errors can reveal underlying cognitiveprocessing difficulties. Within the process of picture naming, three stages can be distinguished (Johnson, Paivio, \& Clark, 1996; Snodgrass \& McCullough, 1986). In the first stage, the depicted object must be correctly identified. After this initial stage of object identification, appropriate names (lemmas) must be activated and selected. In the final stage, articulatory commands are prepared and executed as a lexical response (a phonologically wellstructured lexeme) is generated. Problems during the first stage may occur as a result of general processing difficulties (e.g., inhibitory deficits, visual integration difficulties), sparse semantic representation, or vocabulary problems (in which case, no semantic representation is present in the mental lexicon at all). Problems during name selection may also be the result of sparse semantic representation, but they may also be caused by lexical retrieval problems (word-finding difficulties). Problems during the final stage are generally caused by phonological difficulties (Nation, Marshall, \& Snowling, 2001). When applying a picture naming task, analysis of only naming accuracy is usually not sufficient to determine the exact nature of the problems experienced by the participants. For this reason, it is useful to assess the pattern of naming errors in order to paint a more comprehensive picture.

Picture naming skills have been researched extensively in the case of children with language disorders (Bello, Capirci, \& Volterra, 2004; Dockrell, Messer, \& George, 2001; Lahey \& Edwards, 1999; Nation et al., 2001; Swan \& Goswami, 1997). The pattern of naming errors differs by the type of language problem. As such, the distribution of errors could provide clinicians with an 
indication of the type of language deficit. For example, Lahey and Edwards (1999) found that 6-year-old children with receptive language problems were more likely to make semantic errors, whereas their peers with expressive language problems showed a higher likelihood of phonological errors. Studies on picture naming skills in children with SLI found a lower naming accuracy compared with TD children (e.g., Lahey \& Edwards, 1999; McGregor, Newman, et al., 2002). In the only comprehensive study known to the authors on picture naming skills in children with PLI, subtle differences in performance were visible in a group of 10 children aged 7 and 8, compared with children with SLI (Botting \& ContiRamsden, 1999). However, because the nature of the differences appeared to be qualitative (using atypical strategies for naming) rather than quantitative, with little difference in naming vocabulary, Botting and ContiRamsden suggested that the errors should be qualified as pragmatic. Unfortunately, this study did not compare the nature of naming errors of the PLI children with TD children, nor did it quantify different types of naming errors other than the rate of unusual errors. This category consisted of invented words, words consisting of combining two separate words, and inappropriate alternatives, and was found to be inflated in children with PLI. A more detailed error type analysis may have provided more information regarding the underlying deficits of children with PLI.

In addition to the analysis of naming errors, specific effects of word properties on naming accuracy can also be used as an indicator of underlying deficits. Some researchers have suggested that word frequency has an effect on the way the word is represented in the mental lexicon (Nation et al., 2001). High-frequency words will be represented better than low-frequency words, simply because a child will encounter the low-frequency words to a lesser extent. As such, strong frequency effects can serve as an indicator of a semantic deficit or of the influence of a reduced vocabulary. In contrast, sensitivity to word length can be seen in light of phonological deficits, as longer words place a higher demand on long-term memory (Nation et al., 2001). As to the differential effect of word frequency by disorder, conflicting results have been found (Dockrell et al., 2001). For instance, whereas Wiig, Semel, and Nystrom (1982) found differential effects in the naming of high-frequency words between TD children and children with a language impairment, Wolf (1980) did not. In addition, German (1984) found adverse effects especially in low-frequency words for children with language difficulties and children with learning difficulties. The effect of word length has mainly been established in children with dyslexia. For example, Swan and Goswami (1997) reported on a word length effect for dyslexic children, with no word length effects for any of the children in the control groups; Nation et al. (2001) reported on similar results in their dyslexic sample. The effect of word frequency and word length has not been investigated in children with PLI.

Though research on naming accuracy and naming errors may provide useful information on semantic abilities of children with PLI, naming accuracy by itself offers only a limited view into the mental lexicon. In particular, it is not always possible to determine from the type of naming error whether a semantic representation of a concept is merely sparse, whether the representation is completely lacking, or whether word-finding difficulties are an issue. In order to provide such information, additional tasks can be of use. One possibility is to use some sort of definition task that invites children to provide semantic information regarding the object without requiring the use of the actual name of the object. For the general population, McGregor, Friedman, Reilly, and Newman (2002) studied picture naming skills in relation to semantic representation. Through the use of a drawing and definition task, their results showed that "the degree of knowledge made words more or less vulnerable to retrieval failure" (p. 342) for a group of TD children aged 5. Both drawings and definitions tended to be less accurate and contain less information for pictures that were not correctly named in comparison to pictures that were named correctly. In a subsequent study on semantic representation in 6-year-old children with SLI, they found evidence of limited semantic knowledge and frequent naming errors in this group (McGregor, Newman, et al., 2002). However, research into the way that semantic representations are formed and develop over time is still in its infancy (Brackenbury \& Pye, 2005). A picture naming and definition design has not previously been applied to children with PLI.

In summary, although PLI is characterized as a disorder mainly in the use of language, there is still some doubt about the semantic abilities of these children. Research on the semantic abilities of children with PLI may help to improve transparency of the exact symptoms of these children. Moreover, from a therapeutic standpoint, information on their semantic abilities would be useful in order to provide more accurate treatment. The purpose of the present study was to explore whether young children with pragmatic problems differ from TD peers in their accuracy on a naming and definition task both quantitatively and qualitatively. Specifically, this study attempts to answer the following questions:

1. Do children with PLI show an impaired naming accuracy as compared with TD children?

2. Is naming accuracy differentially affected by word frequency or word length in children with PLI compared with TD children?

3. Do children with PLI show a different pattern of naming errors as compared with TD children? 
4. Do children with PLI show a lower information accuracy in their definitions as compared with TD children?

On the basis of previous research findings, we expected children with PLI to show impaired naming accuracy. Due to the existence of research that finds differential effects of word frequency in children with language disorders compared with TD children, we expected to find a similar differential effect in our PLI sample compared with our TD sample. Regarding word length, we did not expect to find any differential effects, due to the fact that phonological deficits were not expected to be present in our PLI sample. The more interesting issue was whether there was an atypical distribution of error types, as this would provide valuable clues regarding the nature of the problems experienced by children with PLI. We categorized naming errors across the following categories: semantic errors, phonological errors, unrelated errors, omissions and circumlocutions, and made-up words. We did not expect to find differences in phonological errors nor that the additional errors made by the children with PLI would go into any of the other categories. The particular distribution over categories can possibly be used to identify the specific issues experienced by children with PLI.

Although existing research suggests that children with PLI exhibit an unusual pattern of naming errors, we should exclude the possibility that reduced picture naming accuracy could be solely the result of sparse semantic representation and reduced vocabulary, possibly associated with general language delay. Evidence corroborating this hypothesis comes from an early study by Bishop and Rosenbloom (1987), who found that children with pragmatic language problems were considerably delayed in their language development until the age of 5 or 6 . To allow for more definite answers regarding the possibility of reduced vocabulary knowledge, we also included a receptive vocabulary task.

\section{$\overline{\text { Method }}$ \\ Participants}

Participants consisted of 80 TD children (58 boys, 22 girls) and 84 children with PLI (58 boys, 26 girls) recruited from primary schools in the Netherlands. The mean age of both groups was 5;6 ([years;months] $S D$ in months $=3.5$; age range of both groups $=4 ; 11-6 ; 1$ ). The children with PLI were selected on the basis of the pragmatic composite score of the Children's Communication Checklist (CCC; Bishop, 1998). ${ }^{1}$ The CCC is a

\footnotetext{
${ }^{1}$ The presently available CCC-2 resembles the original CCC regarding its content. Differences are found in the phrasing of the questions, the addition of the subscale Semantics, the deletion of the subscale Coherence from the pragmatic composite, and the additional possibilities regarding computation of alternative composite scores.
}

teacher/therapist questionnaire that can be used to identify children with pragmatic language difficulties. Children with a pragmatic composite below the cutoff score of 132 were identified as children with PLI. This cutoff has been identified as a marker for discriminating children with PLI from children with SLI. In order to participate in this study, children could not show serious motor or hearing impairments. Three children in the PLI group were diagnosed as having a speech impairment and were excluded from the analyses. Furthermore, five children were diagnosed with $\mathrm{ADHD}$, and nine were under investigation for an autism spectrum disorder. These children were not excluded. For more information on the participants (prevalence of PLI, teacher opinions, diagnoses, additional behavioral problems), see Ketelaars, Cuperus, Jansonius, and Verhoeven (2009) and Ketelaars, Cuperus, Van Daal, Jansonius, and Verhoeven (2009).

The matching group of TD children (TD group) was selected on the basis of classroom, gender, and age (within 6 months). As a confirmation of their normal development, these children had to attain a pragmatic composite above 140 (lowest score of a TD sample of Bishop and Baird, 2001) and did not show developmental problems as assessed by their teachers. All children spoke Dutch as a first language. Due to excessive shyness of the child or failure of the audio equipment, data of three children in the TD group and six children in the PLI group could not be taken into account.

Teachers were also asked to classify the highest level of completed education of each of the parents on a 4-point scale, where the scores $1-4$ indicate that a parent finished elementary education, lower general secondary education, higher general secondary education, or a college or university degree, respectively. The mean educational level of fathers was $2.97(S D=0.79)$ for the TD group and was $2.68(S D=0.83)$ for the PLI group. This difference between groups did not reach statistical significance, $t(113)=1.90, p=.06$. As for the mean educational level of mothers, a significant difference was not found between the TD group $(M=3.02, S D=0.81)$ and the PLI group $(M=2.59, S D=0.70), t(117)=3.10, p=.01$. For this reason, analyses were conducted both with and without the parental and maternal educational levels as covariates. As parental educational levels were not obtained for all parents, a missing values analysis was performed after it was established using $t$ tests that the naming profiles of the children with missing parental educational levels resembled those of the children whose scores were present.

\section{Background Measures}

Nonverbal reasoning task. Nonverbal reasoning skills were assessed with the Raven Coloured Progressive Matrices (CPM; Raven, 1956). Dutch norms were used to 
convert raw scores to standardized scores, which ranged from 0.5 to 9.5 (Van Bon, 1986), with a mean standardized score of 5 and a standard deviation of 2 .

Receptive vocabulary task. A subtest of the Dutch Language Test for Children (Taaltest Alle Kinderen [TAK]; Verhoeven \& Vermeer, 2001) was administered as a measure of receptive vocabulary. The TAK is a standardized test for 4- to 10-year-olds. In the Receptive Vocabulary subtest, the child is presented with a word and is asked to select the picture illustrating that word out of four pictures. The maximum number of items is 96 , and the task is discontinued after five consecutive errors.

\section{Stimuli}

Picture naming task. The picture naming task consisted of 20 pictures selected from the Dutch adaptation of the Renfrew Word Finding Vocabulary Test (JansoniusSchultheiss, Borgers, DeBruin, \& Stumpel, 2006), which is widely used to assess productive vocabulary in children. Selected pictures were all nouns, either objects or animals. The procedure consisted of asking the child to name the picture (What's this?). Half of the picture names consisted of high-frequency words, and half consisted of low-frequency words. Moreover, half of the picture names were considered short (either one or two syllables with a maximum of five phonemes), and half were considered long (more than two syllables with a minimum of six phonemes). The mean phoneme count was 4.30 for the short words, compared with 8.30 for the long words. Frequency estimates were taken from Schrooten and Vermeer (1994), who made a corpus of children's vocabulary using language from schoolbooks, children's literature, and interactive language in the classroom. High-frequency words were those with a log frequency of 1.5 or higher, whereas low-frequency words were those with a $\log$ frequency between 0.5 and 1.5 . It was decided not to use words with a log frequency below 0.5 because most TD children at this age are not expected to be familiar with these words. The mean log frequency was 1.80 for the high-frequency words, compared with 1.11 for the low-frequency words.

The categorization of error types was based on the categorization of Lahey and Edwards (1999). To circumvent extremely low frequencies for some error types, the different categories were collapsed into the categories of semantic errors, phonological errors, "nonrelated" errors, and a category consisting of omissions and circumlocutions. Semantic errors consisted of errors of a superordinate category, a subordinate category (in which only a specific part of the object was identified), semantically associated responses, responses with a semantic association and a perceptual resemblance, or responses that were semantically associated but perseverative in nature (named earlier in the task). An example of a semantic error would be "aeroplane" for helicopter. Phonological errors consisted of responses in which at least half of the phonemes occurred in the same order, such as rhyming words, plurals, or nonexisting words (e.g., "tigloo" for igloo). Nonrelated errors were those that did not bear either a semantic or phonological relation to the target word. These errors consisted of perceptual errors, in which the response word was similar in shape as the target word, errors that could not be related to the target word in any way, and unrelated perseverative errors, which did not have any relation with the target word but was presented earlier in the task. An example of an unrelated error would be "key" for thermometer. The error category omissions and circumlocutions consisted of "don't know" answers, or instances in which the child talked about the object in the picture but did not name it. Moreover, as literature on PLI reports on a high incidence of "pragmatically strange" words, it was decided to add a category consisting of nonwords (invented words) that do provide an accurate description of the picture ("plantwatergiver" for watering can-both are single words in Dutch).

Regardless of the outcome of the naming task, the children were subsequently asked to define (in the case of objects) the use of the objects or (in the case of animals) define the specific actions of the animal (What can you do with this/What does this do?) without referring to the correct answer. During the elicitation of the definitions, the pictures were present. Information accuracy was scored as nonexistent/erroneous (0); accurate but insufficient, generic information/not core aspect (1); or accurate (2). Coding examples of the definitions are provided in Table 1.

For each child, mean information accuracy scores were computed for all items as well as for the separate categories of correctly named pictures, of semantically related errors, and of omissions and circumlocutions. When semantic errors or omissions and circumlocutions did not occur in a child, the mean information accuracies for these categories were scored as "missing." Information accuracy was computed for the nonrelated error category; however, this turned out to be close to zero in most

Table 1. Coding examples of information accuracy in the definition task.

\begin{tabular}{|c|c|c|}
\hline Naming category & Score & Example \\
\hline Accurate definition & 2 & $\begin{array}{l}\text { "you can make music with it" (guitar) } \\
\text { "it can make a hole in the wall" (drill) }\end{array}$ \\
\hline $\begin{array}{l}\text { Accurate but } \\
\text { insufficient/generic }\end{array}$ & 1 & $\begin{array}{l}\text { "it can walk" (caterpillar) } \\
\text { "you can use it to look" (binoculars) }\end{array}$ \\
\hline Nonexistent/erroneous & 0 & $\begin{array}{l}\text { "don't know" } \\
\text { "it can saw" (drill) } \\
\text { "you can catch fish with it" (anchor) }\end{array}$ \\
\hline
\end{tabular}


cases, and therefore this category was not included in the subsequent analyses. For the nonwords error category and for the phonological error category, the number of samples was too low to provide for meaningful results, so these categories were excluded from the subsequent analyses as well.

Though we explicitly asked the children to describe functions (through the use of the question: What can you do with this/what does this do?), definitions were also scored for physical, locative, evaluative, or categorical information units (McGregor, Newman, et al., 2002). Moreover, the use of nonverbal definition strategies such as sounds (e.g., making a sound of a guitar) or movements as replacements for verbal strategies were also scored but were assigned an information accuracy of zero.

When the naming and defining conditions did not lead to accurate naming, in the Dutch adaptation of the Renfrew Word Finding Vocabulary Test, children were given semantic cues (e.g., for caterpillar, "it changes into a butterfly"). The individual effect of semantic cues was computed by summing the number of correct responses after a semantic cue was provided and dividing it by the total number of semantic cues given.

For interrater reliability purposes, a subsample of 39 children (a mixed sample of TD children and PLI children) was also coded independently by a second coder. Point-to-point agreement between the coders was $94 \%$ (range $=85 \%-100 \%$ ) for the naming errors. For the definition accuracy, point-to-point agreement was $88 \%$ (range $=70 \%-100 \%)$.

\section{Procedure}

This study took place in the context of a wider study on the skills and deficits of children with PLI, which aims to shed more light on the diagnostic status of PLI. Children were tested at their schools in two sessions of approximately $50 \mathrm{~min}$ each. Upon entering the room, the children were first familiarized with the situation and with the experimenter. Tasks were completed in a fixed order.

\section{$\overline{\text { Results }}$ \\ Nonverbal Reasoning}

Regarding nonverbal reasoning skills, the TD group attained a mean standardized score of $6.22(S D=1.76)$ on Raven's CPM, whereas the PLI children attained a mean standardized score of $5.03(S D=2.07)$. The difference between both groups reached significance, $t(151)=$ $3.84, p<.001$. Comparing the standardized scores of the PLI group with the original sample showed a normal mean score, whereas the TD group showed a slightly higher than expected score. This might be caused by the fact that our TD children were screened for developmental problems. Because differences in nonverbal reasoning skills were found, further analyses were carried out both with and without nonverbal reasoning as a covariate.

\section{Receptive Vocabulary}

Table 2 shows the means and standard deviations of the PLI group in comparison to the TD group. A $t$ test yielded a significant difference, in favor of the TD children, $t(145.32)=6.59, p<.001$. The difference remained significant after controlling for nonverbal reasoning skills and parental educational levels.

\section{Naming Accuracy}

Means and standard deviations of naming accuracy are shown in Table 2. To assess differences in naming accuracy, we performed a 2 (group) $\times 2$ (word length) $\times$ 2 (word frequency) analysis of variance (ANOVA), with word length and word frequency as within-subjects factors and group as a between-subjects factor. According to the repeated measures ANOVA, there was a significant main effect of word frequency, $F(1,151)=284.35, p<.001$, $\eta_{\mathrm{p}}^{2}=.65$; word length, $F(1,151)=30.45, p<.001, \eta_{\mathrm{p}}^{2}=.17$; and group, $F(1,151)=31.41, p<.001, \eta_{\mathrm{p}}^{2}=.17$. The Group $\times$ Word Frequency interaction was nonsignificant, $F(1,151)=3.00, p=.09, \eta_{\mathrm{p}}^{2}=.02$. Despite relatively high performance, the PLI group did show a lower overall accuracy rate compared with the TD group. All groups performed better on high-frequency words and short words. To ensure differences were not caused by differences in nonverbal reasoning skills, we repeated the analysis with the Raven's CPM scores and parental educational levels as covariates. All main effects remained significant.

Table 2. Means and standard deviations on naming accuracy and receptive vocabulary across groups.

\begin{tabular}{lccccc}
\hline & \multicolumn{2}{c}{ TD group } & & \multicolumn{2}{c}{ PLI group } \\
\cline { 2 - 3 } \cline { 6 - 6 } \multicolumn{1}{c}{ Variable } & $M$ & SD & & $M$ & SD \\
\hline $\begin{array}{l}\text { Receptive vocabulary } \\
\text { Naming accuracy }\end{array}$ & 65.10 & 10.64 & & 52.45 & 12.39 \\
HF-S & & & & & \\
HF-L & 4.32 & 0.79 & & 4.01 & 0.90 \\
LF-S & 4.19 & 0.80 & & 3.59 & 0.93 \\
LF-L & 3.36 & 0.93 & & 2.70 & 1.17 \\
\cline { 6 - 7 } & 2.96 & 0.99 & & 2.21 & 1.14 \\
\hline
\end{tabular}

Note. $\mathrm{TD}=$ typically developing; $\mathrm{PLI}=$ pragmatic language impairment; $\mathrm{HF}=$ high-frequency condition; $\mathrm{S}=$ short-word condition; $\mathrm{L}=$ long-word condition; LF = low-frequency condition. 


\section{Error Type}

We performed analyses of error types using absolute numbers of errors as well as the proportions of errors of a specific type relative to the total numbers of errors. As overall error rates were low, the errors of the different stimulus conditions (word frequency and word length) were collapsed. Table 3 shows the absolute numbers and relative proportions of errors for the different groups. The most common errors for both the PLI group and the TD group were found in the category omissions and circumlocutions (63\% of the errors of the TD group and 53\% of the errors of the PLI group), followed by semantic errors (22\% of the errors of the TD group and $28 \%$ of the errors of the PLI group). The incidence of phonological errors was close to zero.

We analyzed differences in the distribution of error types by means of one-way ANOVAs using the absolute numbers of errors and the error-type proportion. As Table 3 shows, children with PLI showed a higher total rate of errors. Furthermore, they showed higher rates of semantic errors, of errors not related to the target word, and of omissions and circumlocutions. They did not show a higher rate of phonological errors or made-up words. Concerning the proportions of errors, the only significant difference was found within the nonrelated category. Children with PLI responded significantly more often with a word not related to the target word (e.g., "tap" instead of microphone, "grass" instead of arrow). To find whether the children with PLI showed a preponderance of specific errors in the nonrelated category, we further subdivided this category into object identification errors, of which the named objects showed a visual resemblance to the target object (misperceptions), naming errors that could really not be related to the target word (possibly nonwords), and naming errors that were considered perseverative. Most of the nonrelated naming errors of the PLI group consisted of misperceptions with a visual resemblance (59\% of all nonrelated errors), followed by truly unrelated errors ( $31 \%$ of all nonrelated errors). The TD group showed a similar pattern: $61 \%$ of all nonrelated errors consisted of misperceptions, and $23 \%$ of all nonrelated errors consisted of truly unrelated errors. $T$ tests showed that children with PLI responded with a higher number of misperceptions, $t(127.37)=-3.47$, $p<.01$, and truly unrelated errors, $t(110.40)=-3.23$, $p<.01$. We performed the same analyses adding nonverbal reasoning and parental educational levels as covariates. Most reported differences remained significant, with the exception of the total number of omissions and circumlocutions, which was no longer significant. Interestingly, the difference in proportion of omissions and circumlocutions between both groups was significant after adding the covariates. The TD children showed a higher proportion of omissions and circumlocutions $(p=.05)$. It should be noted that, significant differences between groups notwithstanding, the large standard deviations also suggest sizable differences between children within either group.

Shapiro-Wilks's tests indicated violation of the normality assumption of the ANOVA procedure. We reanalyzed the data using a nonparametric procedure in order to verify that the results were not artifacts of this assumption

Table 3. Means and standard deviations on number of errors and proportion of errors across category for both groups.

\begin{tabular}{|c|c|c|c|c|c|c|c|c|c|c|}
\hline \multirow[b]{2}{*}{ Variable } & \multicolumn{4}{|c|}{ TD group } & \multicolumn{4}{|c|}{ PL group } & \multirow[b]{2}{*}{$\boldsymbol{F}$} & \multirow[b]{2}{*}{$p$} \\
\hline & $M$ & $S D$ & Mdn & Range & $M$ & $S D$ & Mdn & Range & & \\
\hline \multicolumn{11}{|l|}{ Semantic errors } \\
\hline Number of semantic errors & 1.19 & 1.40 & 1 & $0-5$ & 2.05 & 1.65 & 2 & $0-7$ & 12.08 & $<.001$ \\
\hline Number of phonological errors & 0.12 & 0.36 & 0 & $0-2$ & 0.18 & 0.53 & 0 & $0-3$ & 0.83 & .36 \\
\hline Proportion of phonological errors & .03 & .09 & 0 & $0-.67$ & .02 & .06 & 0 & $0-.25$ & 0.04 & .84 \\
\hline \multicolumn{11}{|l|}{ Nonrelated errors } \\
\hline Number of no-relation errors & 0.36 & 0.74 & 0 & $0-4$ & 1.01 & 1.09 & 1 & $0-4$ & 18.63 & $<.001$ \\
\hline Proportion of no-relation errors & .07 & .13 & 0 & $0-.50$ & .13 & .14 & .12 & $0-.50$ & 9.39 & $<.001$ \\
\hline \multicolumn{11}{|l|}{ Made-up words } \\
\hline Number of made-up words & 0.22 & 0.45 & 0 & $0-2$ & 0.24 & 0.49 & 0 & $0-2$ & 0.05 & .83 \\
\hline Proportion of made-up words & .05 & .11 & 0 & $0-.50$ & .04 & .09 & 0 & $0-.05$ & 0.75 & .39 \\
\hline
\end{tabular}


violation. The outcomes of these Mann-Whitney $U$ tests were largely similar to the original ANOVA results, showing additional significant differences between both groups on the measures' proportion of semantic errors $(p=.04)$ and proportion of omissions and circumlocutions $(p=.04)$, which were elevated in the children with PLI.

\section{Definition Task}

For each child, we computed the mean information accuracy over all items. On average, the TD group had a mean information accuracy per defined picture of 1.50 points $(S D=0.24)$. As the maximum score of information accuracy is 2.00 , the mean information accuracy of the TD group indicates a fairly good understanding of the concepts. The PLI group had an overall lower, yet still relatively high, information accuracy per picture of 1.27 points $(S D=0.25)$, a difference that reached significance, $t(153)=5.79, p<.001$. The difference remained significant after controlling for nonverbal reasoning skills and parental educational levels. However, because the PLI group had an overall lower naming accuracy, lower information accuracy could be expected. We therefore computed separate information accuracy scores for every child for correct naming responses, for semantic errors, and for omissions and circumlocutions. Although this provides us with a possibility to compare groups on information accuracy across similar error categories, one needs to take into account that results on the betweengroup comparison should be treated carefully, as the comparisons within the naming categories are not based on the same items. The mean information accuracy in the definition condition for correct naming responses, semantic errors, and omissions and circumlocutions are shown in Table 4.

We conductd a repeated measures ANOVA to reveal differences between groups and error categories, with the different naming categories as a within-subjects factor and group as a between-subjects factor. The main effect of naming category was significant, $F(2,84)=95.61$, $p<.001, \eta_{\mathrm{p}}{ }^{2}=.70$. Post hoc comparisons using Bonferroni adjustment showed that information accuracy was higher for the accurate naming category compared with the semantic and omissions/circumlocution error categories

Table 4. Means and standard deviations on information accuracy across groups.

\begin{tabular}{lcccccc} 
& \multicolumn{2}{c}{ TD group } & & \multicolumn{2}{c}{ PLI group } \\
\cline { 2 - 3 } \cline { 6 - 7 } \multicolumn{1}{c}{ Naming category } & M & SD & & M & SD \\
\hline Accurate naming category & 1.69 & 0.18 & & 1.56 & 0.26 \\
Semantic naming error category & 1.08 & 0.75 & & 0.85 & 0.65 \\
Omission and circumlocution & 0.80 & 0.63 & & 0.65 & 0.55 \\
\hline
\end{tabular}

( $p \mathrm{~s}<.001)$. The main effect of group was not significant, $F(1,85)=2.46, p=.12, \eta_{\mathrm{p}}^{2}=.03$. However, this might be related to the fact that many cases were deleted because of missing values. These missing values consisted of cases in which no errors of a certain category were made. Therefore, we performed three separate ANOVAs, with group as an independent variable and information accuracy in the three respective naming categories as dependent variables. Results showed that the PLI group showed a lower information accuracy in the accurate naming category, $F(1,150)=13.22, p<.001$. Although there was a difference in information accuracy in the semantic error category, it did not reach statistical significance, $F(1,93)=2.57, p=.11$. The difference in information in the omission and circumlocutions category did not reach significance either, $F(1,137)=2.32, p=.13$. Qualitative analysis on the definitions given by the children in the PLI group revealed a tendency to use pragmatically odd words in their definitions, words that do exist but could be considered inappropriate in the given context (e.g., a drill "cuts" through wood, a snail "sleds" over the floor), which led to lower scores on overall information accuracy. All differences remained similar when nonverbal reasoning and parental educational levels were added as a covariate.

The use of other definition strategies (categorical, evaluative, locative) did not occur frequently and did not differ between both groups, although after controlling for nonverbal reasoning skills and parental educational levels, the TD group did make more frequent use of categorical information. The use of movement and sounds as means of defining, though infrequent, did show a trend, with the PLI group showing a higher rate (.26 vs. .08), $t(92.49)=-1.92, p=.06$. The difference disappeared when nonverbal reasoning and parental educational levels were controlled for.

\section{Effect of Semantic Cues}

We computed the effect of semantic cues by summing the number of correct responses after semantic cues and dividing it by the total number of semantic cues given. Both groups benefited roughly equally from the use of semantic cues, $t(149)=0.34, p=.73$. The TD children correctly named an additional $10.02 \%$ of the pictures, whereas the PLI group correctly named an additional $9.22 \%$ of the pictures. A similar picture was visible after we controlled for nonverbal reasoning and parental educational levels.

\section{Conclusions and Discussion}

The main purpose of the present study was to clarify the extent to which children with PLI show semantic 
problems in a picture naming task as compared with TD children. This research intends to improve upon earlier research into the semantic abilities of children with PLI, which unfortunately compared the children to control groups consisting of children with SLI, which made it difficult to draw conclusions on the actual semantic abilities of these children.

In general, as hypothesized, we found evidence for semantic problems in this population: The PLI group showed a reduced receptive vocabulary in combination with a lower rate of picture naming accuracy compared with TD children. The picture naming accuracy of both the PLI group and the TD children was affected by either word length or word frequency; short words and highfrequency words were named more accurately than were longer and low-frequency words.

With regard to picture naming error types, the performance of the PLI group was characterized by higher error rates across most error categories. However, when the proportion of errors relative to the total number of errors was considered, only a difference in the category of nonrelated errors was discernable: The PLI group showed twice as high a proportion of these errors. The relative proportion of other errors such as phonological or semantic errors was comparable to those of the TD group (i.e., error rates were equally elevated for all error types).

In the definition task, we found lower information accuracy for the PLI group compared with the TD children: Children with PLI showed more difficulty defining the function (or in the case of animals, the abilities) of the object displayed in the picture. This difference held for both the entire test as well as within the accurate picture naming response category. They did show a similar pattern across the picture naming response categories compared with the TD group, in that their information accuracy was higher in the correctly named pictures than for the semantic error category and omissions/ circumlocutions error categories. Qualitative analysis of the definitions of the PLI group revealed a tendency to use pragmatically odd words in their definition, words that do exist but that could be considered inappropriate in the given context. Moreover, they showed a slightly higher frequency of movement and sounds, a finding that partly matches results found in a study on the use of gestures in children with SLI (Evans, Alibali, \& McNeil, 2001). The use of gestures could possibly be interpreted as a compensatory strategy. Interestingly, after controlling for nonverbal reasoning skills and parental educational levels, the difference between both groups disappeared.

As a final result, we found that although children with PLI benefited from semantic cues, their performance did not reach normal levels after semantic cues were given, which is partly due to the similar beneficial effect for the TD children. This finding is comparable to findings concerning phonological and semantic cues by Gray (2005). She found beneficial effects for both phonological and semantic cues in 5-year-old children with SLI. Although semantic cues seemed to be of more use in a receptive task, phonological cues led to a higher result in a production task for the SLI group. However, neither semantic cues nor phonological cues led to a naming level consistent with that of their TD peers. The results of our study suggest that retrieval problems are not an issue in our PLI sample but that semantic representation might actually be lacking entirely.

As stated in the introduction, cognitive models on naming distinguish three stages. Specific effects of word properties as well as specific patterns of naming errors can provide information on possible stages at which problems occur. We hypothesized that the rate of phonological errors would not be elevated in our PLI sample, which indeed was the case. This suggests that problems do not occur during the stage at which articulatory commands are prepared. As to the possibility of elevated error levels in the other stages, existing research was not clear. The evidence we found for naming errors in children with PLI suggest possible deficits in both the object identification and name selection stages. Compared with TD children, a large proportion of the errors of the PLI group consisted of nonrelated responses, which could have been the result of erroneous object identification. Interestingly, in a subanalysis of these nonrelated errors, we found a statistically significant predisposition of the PLI group to identify pictures on the basis of a shared physical resemblance, a finding that does not seem to correspond with evidence regarding naming errors in other children with language disorders (Nation et al., 2001). In the case of our PLI group, it seemed that small contextual cues concerning the real identity of the picture were not taken into account, causing faulty object identification. One possible explanation of this phenomenon can be found in the central coherence theory (Frith, 1989). A deficit in central coherence, the ability to integrate information often at the expense of details, is widely used as a theory in the explanation of autistic spectrum disorders. In the debate on the validity of PLI as a disorder, an overlap with autism spectrum disorders has been suggested. The finding of possible subtle central coherence problems in our PLI group is consistent with such an overlap. However, this is just a hypothesis; it would be interesting to investigate whether children with PLI do indeed exhibit subtle central coherence problems and, if so, whether these problems are related to their naming errors. Alternatively, ambiguity of some of the illustrations might have led to visual misperceptions. This is corroborated by the finding that most of the unrelated errors of the TD children also consisted of these visual misperceptions. However, 
the children in the PLI group did seem more prone to this effect.

Evidence for problems was found during the second stage in picture naming; that is, name selection includes a lower semantic representation of known words, as evidenced by a lower definition accuracy, and a higher frequency of semantic errors. However, semantic errors are often reported to be the most frequent error type, and claims regarding semantic problems should not be made without caution. The combination of a higher proportion of nonrelated errors, sparse semantic representation, and lower receptive vocabulary seems rather consistent with a general language delay (with a reduced vocabulary knowledge), as suggested by Bishop and Rosenbloom (1987). In theory, the lower definition accuracy of the known words could simply reflect this general language delay through the reduced ability of the subjects to express the semantic knowledge that they possess. Our sample consisted of 5-year-olds, which is a younger age compared with most other research on this subject. It might very well be that the children with PLI gradually catch up with their TD peers in the area of vocabulary knowledge, whereas pragmatic deficits may be more persistent in nature. That would explain why evidence for lower naming accuracy was not found in other study samples. Unfortunately, a comprehensive design was not used in studies in this area, which investigated naming accuracy, naming errors, and broader semantic representation. As such, it is difficult to compare our results with the existing research.

Although the results of this study shed more light on the semantic abilities of children with pragmatic language problems, some limitations need to be mentioned. Our PLI group, based on a community sample, was selected using the CCC. Though the CCC is a questionnaire designed to assess PLI, one should be careful to diagnose a child solely on the basis of this questionnaire. Recent findings do suggest that the CCC is viable as a screening instrument (Ketelaars, Cuperus, Van Daal, et al., 2009). Second, it would be interesting to relate the semantic problems experienced in this picture naming task to semantic abilities in a more natural setting. The picture naming task used in this study could be regarded as an artificial task, and questions remain as to the semantic problems of PLI children in daily conversations. However, findings by McGregor (1997) reveal similar semantic problems in a picture naming task as well as a narrative task, which suggest that the semantic problems that occur in a picture naming task are a genuine reflection of semantic problems in a more natural setting. In addition, the items of the naming tasks were nouns. It remains to be seen whether the results would also extend to other semantic word classes. It should also be noted that elicitation of the definitions occurred in the presence of the picture and without providing the correct answer, which means that the definitions were based on knowledge of the depicted objects and not necessarily of the target word. Removing the picture and providing the correct answer when asking for a definition as done by McGregor, Newman, et al. (2002), for example, may yield different results. As a final limitation, it would have been valuable to include a drawing task, which investigates semantic representation in a nonlinguistic fashion.

In conclusion, this study yields strong indications that semantic problems do occur in pragmatically impaired children and that earlier conclusions regarding the absence of such problems in children with PLI may have been premature. This implies that clinicians need to consider the possible presence of subtle semantic difficulties in children who experience pragmatic problems. Moreover, the present study indicates that the semantic problems are not uniform, as indicated by large standard deviations in many of the results, which implies that care is required to correctly identify the problem areas of individual children. Picture naming tasks might be of assistance here, perhaps also combined with a definition task. However, because standard deviations are large, the practical use would have to involve qualitative rather than quantitative analysis.

\section{References}

Bello, A., Capirci, O., \& Volterra, V. (2004). Lexical production in children with Williams syndrome: Spontaneous use of gesture in a naming task. Neuropsychologia, 42, 201-213.

Bishop, D. V. M. (1998). Development of the Children's Communication Checklist (CCC): A method for assessing qualitative aspects of communicative impairment in children. Journal of Child Psychology and Psychiatry, 39, 879-891.

Bishop, D. V. M. (2000). Pragmatic language impairment: A correlate of SLI, a distinct subgroup, or part of the autistic continuum? In L. B. Leonard \& D. V. M. Bishop (Eds.), Speech language impairments in children: Causes, characteristics, intervention and outcome (pp. 99-113). Hove, England: Psychology Press.

Bishop, D. V., \& Adams, C. (1989). Conversational characteristics of children with semantic-pragmatic disorder: II. What features lead to a judgement of inappropriacy? British Journal of Disorders of Communication, 24, 241-263.

Bishop, D. V. M., \& Baird, G. (2001). Parent and teacher report of pragmatic aspects of communication: Use of the Children's Communication Checklist in a clinical setting. Developmental Medicine and Child Neurology, $43,809-818$.

Bishop, D. V. M., \& Norbury, C. F. (2002). Exploring the borderlands of autistic disorder and specific language impairment: A study using standardised diagnostic 
instruments. Journal of Child Psychology and Psychiatry, 43, 917-929.

Bishop, D. V. M., \& Rosenbloom, L. (1987). Classification of childhood language disorders. In W. Yule \& M. Rutter (Eds.), Language development and disorders: Clinics in developmental medicine (pp. 16-41). London, England: MacKeith Press.

Botting, N., \& Conti-Ramsden, G. (1999). Pragmatic language impairment without autism: The children in question. Autism, 3, 371-396.

Brackenbury, T., \& Pye, C. (2005). Semantic deficits in children with language impairments: Issues for clinical assessment. Language, Speech, and Hearing Services in Schools, $36,5-16$.

Brook, S. L., \& Bowler, D. M. (1992). Autism by another name? Semantic and pragmatic impairments in children. Journal of Autism and Developmental Disorders, 22, 61-81.

Camarata, S. M., \& Gibson, T. (1999). Pragmatic language deficits in attention-deficit hyperactivity disorder (ADHD). Mental Retardation and Developmental Disabilities, 5, 207-214.

Dockrell, J. E., Messer, D., \& George, R. (2001). Patterns of naming objects and actions in children with word finding difficulties. Language and Cognitive Processes, 16, 261-286.

Evans, J. L., Alibali, M. W., \& McNeil, N. M. (2001). Divergence of verbal expression and embodied knowledge: Evidence from speech and gesture in children with specific language impairment. Language and Cognitive Processes, $16,309-331$.

Frith, U. (1989). Autism: Explaining the enigma. Oxford, England: Blackwell.

German, D. J. (1984). Diagnosis of word-finding disorders in children with learning disabilities. Journal of Learning Disabilities, 17, 353-358.

Geurts, H. M., Verté, S., Oosterlaan, J., Roeyers, H., Hartman, C. A., Mulder, E. J., ... Sergeant, J. A. (2004). Can the Children's Communication Checklist differentiate between children with autism, children with $\mathrm{ADHD}$, and normal controls? Journal of Child Psychology and Psychiatry, 45, 1437-1453.

Gray, S. (2005). Word learning by preschoolers with specific language impairment: Effect of phonological or semantic cues. Journal of Speech, Language, and Hearing Research, $48,1452-1467$.

Jansonius-Schultheiss, K., Borgers, M., DeBruin, B., \& Stumpel, H. (2006). Renfrew's language scales Dutch adaptation. Amsterdam, the Netherlands: Pro-education, HvA.

Johnson, C. J., Paivio, A., \& Clark, J. M. (1996). Cognitive components of picture naming. Psychological Bulletin, 120, $113-139$.

Ketelaars, M. P., Cuperus, J. M., Jansonius, K., \& Verhoeven, L. (2009). Pragmatic language impairment and associated behavioural problems. International Journal of Language and Communication Disorders, 45, 204-214.

Ketelaars, M. P., Cuperus, J. M., Van Daal, J., Jansonius, K., \& Verhoeven, L. (2009). Screening for pragmatic language impairment: The potential of the Children's Communication Checklist. Research in Developmental Disabilities, 30, 952-960.

Lahey, M., \& Edwards, J. (1999). Naming errors of children with specific language impairment. Journal of Speech, Language, and Hearing Research, 42, 195-205.

McGregor, K. K. (1997). The nature of word-finding errors of preschoolers with and without word-finding deficits. Journal of Speech, Language, and Hearing Research, 40, 1232-1244.

McGregor, K. K., Friedman, R. M., Reilly, R. M., \& Newman, R. M. (2002). Semantic representation and naming in young children. Journal of Speech, Language, and Hearing Research, $45,332-346$.

McGregor, K. K., Newman, R. M., Reilly, R. M., \& Capone, N. C. (2002). Semantic representation and naming in children with specific language impairment. Journal of Speech, Language, and Hearing Research, 45, 998-1014.

Miniscalco, C., Hagberg, B., Kadesjö, B., Westerlund, M., \& Gillberg, C. (2007). Narrative skills, cognitive profiles and neuropsychiatric disorders in 7-8-year-old children with late developing language. International Journal of Language and Communication Disorders, 42, 665-681.

Nation, K., Marshall, C. M., \& Snowling, M. J. (2001) Phonological and semantic contributions to children's picture naming skill: Evidence from children with developmental reading disorders. Language and Cognitive Processes, 16, 241-259.

Rapin, I., \& Allen, A. (1983). Developmental language disorders: Nosological considerations. In U. Kirk (Ed.), Neuropsychology of language, reading and spelling (pp. 155-184). London, England: Academic Press.

Raven, J. C. (1956). Guide to using the Coloured Progressive Matrices. London, England: H.K. Lewis.

Sahlén, B., \& Nettelbladt, U. (1993). Context and comprehension: A neurolinguistic and interactional approach to the understanding of semantic-pragmatic disorder. European Journal of Disorders of Communication, 28, 117-140.

Schrooten, W., \& Vermeer, A. (1994). Woorden in het basisonderwijs. 15.000 woorden aangeboden aan leerlingen [Words in elemenary school. 15.000 words presented to students]. Tilburg, the Netherlands: Tilburg University Press.

Snodgrass, J. G., \& McCullough, B. (1986). The role of visual similarity in picture categorization. Journal of Experimental Child Psychology: Learning, Memory, and Cognition, 12, 147-154.

Swan, D., \& Goswami, U. (1997). Picture naming deficits in developmental dyslexia: The phonological representations hypothesis. Brain and Language, 56, 334-353.

Van Balkom, H., \& Verhoeven, L. (2004). Pragmatic disability in children with specific language impairments. In L. Verhoeven \& H. van Balkom (Eds.), Classification of developmental language disorders: Theoretical issues and clinical implications (pp. 283-305). Mahway, NJ: Lawrence Erlbaum Associates.

Van Bon, W. H. J. (1986). Raven's Coloured Progressive Matrices: Manual of Dutch norms. Lisse, the Netherlands: Swets \& Zeitlinger 
Verhoeven, L., \& Vermeer, A. (2001). Taaltest Alle Kinderen (TAK) [Language test for all children]. Arnhem, the Netherlands: CITO.

Wiig, E., Semel, E., \& Nystrom, L. (1982). Comparison of rapid naming abilities in language-learning disabled and academically achieving eight-year-olds. Language, Speech, and Hearing Services in Schools, 13, 11-23.

Wolf, M. (1980). The word-retrieval process and reading in children and aphasics. Children's Language, 3, 437-490.
Received June 7, 2009

Revision received November 16, 2009

Accepted May 24, 2010

DOI: 10.1044/1092-4388(2010/09-0116)

Contact author: Mieke Pauline Ketelaars, Behavioural Science Institute, Department of Special Education, Radboud University Nijmegen, P.O. Box 9104, $6500 \mathrm{HE}$ Nijmegen, the Netherlands. E-mail: M.Ketelaars@pwo.ru.nl. 